Schmerz 2012 $\cdot 26: 367-368$

DOI 10.1007/s00482-012-1213-x

(C) Deutsche Schmerzgesellschaft e.V. Published by Springer-Verlag -

all rights reserved 2012

\author{
W. Meissner ${ }^{1,2}$ \\ ${ }^{1}$ Klinik für Anästhesiologie und Intensivtherapie, Universitätsklinikum Jena \\ 2 Klinik für Innere Medizin II, Abteilung Palliativmedizin, Universitätsklinikum Jena
}

\section{Qualität in der (Akut-)Schmerztherapie} Plädoyer für einen Paradigmenwechsel
Böhmer et al. [1] berichten in diesem Heft über eine Befragung von Kliniken, die sich im Rahmen des Projekts Qualitätsmanagement Akutschmerztherapie des TÜV Rheinland zertifizieren ließen. Dieser Artikel beleuchtet exemplarisch die Stärken, Probleme und Herausforderungen einer Qualitätsverbesserung in der Schmerzmedizin und darüber hinaus.

Maßnahmen zur Qualitätssicherung und -verbesserung in Deutschland sind traditionell geprägt durch die checklistenartige Erfassung von Struktur- und Prozessindikatoren, von denen man annimmt, dass sie einen Zusammenhang mit dem Ergebnis des Gesamtprozesses haben - ein Konzept, dass aus der Wirtschaft stammt und dazu dient, die Qualität von Zulieferern und deren Produkten berechenbarer zu machen. Dieser Ansatz besitzt auch in der Medizin große Vorteile: Struktur- (z. B. das Vorhandensein von Akutschmerzdiensten oder Pumpen zur patientenkontrollierten Analgesie) oder Prozessparameter (z. B. eine regelmäßige Schmerzmessung oder Patienteninformation) lassen sich in der Regel relativ leicht und kostensparend erheben und sind gut $\mathrm{zu}$ quantifizieren. Relevant für das Behandlungsergebnis sind sie jedoch nur dann, wenn sie nachweisbar in einem engen Verhältnis zum Prozessergebnis stehen - was auch immer die Beteiligten darunter verstehen (s. unten).

Dies lässt sich gut am Beispiel der Routineschmerzmessung und -dokumentation demonstrieren, die geradezu mantrahaft in allen Leitlinien als einer der wichtigsten Prozesse zur Qualitätsverbesse- rung in der Schmerztherapie empfohlen und auch in der vorliegenden Arbeit als ein geforderter - und zu 100\% erfüllter Qualitätsindikator zitiert wird. Die Annahme, Schmerzmessung sei mit Therapiequalität assoziiert, erscheint einerseits „logisch“, da man etwas nur verbessern kann, wenn es gemessen wird. Andererseits beruht sie auf einer bemerkenswert geringen Zahl von - teilweise sehr alten - klinischen Studien. Die in diesem Zusammenhang am häufigsten - und in der deutschen Leitlinie einzige - zitierte Arbeit ist monozentrisch und nicht randomisiert. Sie beschreibt eine Verringerung der Schmerzintensität nach der Einführung von Schmerzmessung in einem Waliser Krankenhaus Anfang der 90erJahre [2].

Andererseits konnte mehrfach gezeigt werden, dass die Einführung einer Routineschmerzmessung nicht automatisch eine Verbesserung der Schmerztherapie zur Folge hat [3], sondern - gerade in $\mathrm{Zu}$ sammenhang mit einer Zertifizierung möglicherweise sogar zu einer Patientengefährdung führt [4]. Zudem unterschied sich die Ergebnisqualität weder in deutschen noch in internationalen Registerdaten auch nur tendenziell zwischen $\mathrm{Pa}$ tienten mit und ohne Routineschmerzmessung [5, 6]. Die Ursachen hierfür sind wahrscheinlich vielfältig. Beispielsweise führen fehlende Behandlungsalgorithmen oder Therapienebenwirkungen $\mathrm{zu}$ einem unzureichenden Behandlungserfolg; andererseits können erfahrene Pflegekräfte auch ohne Schmerzdokumentation Schmerzen ihrer Patienten erfassen und behandeln. Damit ereilt den Qualitätsindikator Schmerzmessung jedoch ein ähnliches Schicksal wie viele andere Surrogatparameter in der Medizin, deren Indikatoreigenschaften aus kleinen prospektiven Studien sich nicht im klinischen Alltag reproduzieren lassen.

Dies bedeutet für die tägliche Praxis natürlich nicht, die Routineschmerzmessung einzustellen, macht aber deutlich, dass sie nicht als Qualitätsindikator verwendet werden sollte, und mahnt zu äußerster Vorsicht bei der Annahme, isolierte Strukturen oder Prozesse führten „automatisch“ zu einer Verbesserung der Ergebnisqualität. Der einzige - und naheliegende - Ausweg aus diesem Dilemma ist die direkte Messung der Ergebnisqualität - ohne Umweg über ein Surrogat. Dies empfehlen im Übrigen auch Böhmer et al. Im Bereich der Schmerzmedizin bedeutet das, zum Äußersten zu schreiten und auch Patienten zu befragen oder modern „patient-reported outcomes“ zu erheben. Dies muss jedoch unter standardisierten Bedingungen erfolgen - die wenigsten Einrichtungen erheben bisher Routinedaten im klinischen Alltag in einer Qualität, die eine multizentrische Vergleichbarkeit erlauben würde.

Auf den ersten Blick erstaunt es, dass wirklich ergebnisorientierte Qualitätssicherung oft viel schwerer einen Weg in die Kliniken findet als Verfahren, die auf Strukturen und Prozesse fokussieren. Außerhalb der Schmerzmedizin ist dieses Phänomen sogar noch viel deutlicher $\mathrm{zu}$ beobachten. Einige der Gründe deuten auch die Autoren an: Immer noch nutzen 
Kliniken und ihre Träger Zertifizierungen vorrangig als (vermeintliches) Marketinginstrument, zur Kostenreduktion oder zur formalen Erfüllung gesetzlicher Auflagen. Auch dann können struktur- und prozessorientierte Zertifizierungsprozesse zwar hilfreich sein, beispielsweise, wenn sie die Vorhaltung von Minimalstrukturen oder die Mitwirkung aller Prozessbeteiligten in einer Klinik erzwingen. Ein endogenes Interesse an einer wirklichen Verbesserung der Ergebnisqualität drückt dies jedoch nicht immer aus. Vielleicht ist es kein $\mathrm{Zu}$ fall, dass einige der großen privaten Klinikbetreiber auch in der Schmerzmedizin inzwischen immer stärker auf outcomeorientierte Verfahren zum Qualitätsmanagement setzt und Ergebnisqualität in das Informationsportal „Qualitätskliniken.de“ auch als "patient-reported outcomes" (in Form einer stichprobenhaften Patientennachbefragung) aufgenommen wurde.

Aber was ist eigentlich gute Qualität in der Schmerzmedizin? Es wurde bereits angedeutet, dass die alleinige Orientierung an Schmerzintensität bzw. an ihrer Reduktion fragwürdig und eventuell sogar gefährlich sein kann, da man sich damit einseitig auf eine schmerzmedizinische Unterversorgung konzentriert, eine Übertherapie jedoch unbeachtet bleiben bzw. sogar gefördert werden könnte. Ähnlich kritisch sind die - glücklicherweise mittlerweile verlassene - Propagierung von „schmerzfreien“ Institutionen, aber auch die bisweilen dogmatische Fixierung anästhesiologischer Akutschmerzdienste auf maximale Schmerzreduktion nach Operationen zu bewerten - ignorierend, dass Übelkeit, motorische Beeinträchtigung, Blasenkatheterpflicht oder Autonomieverlust durch apparative Therapieverfahren den Nutzen der Schmerzreduktion zunichtemachen können.

In einem kürzlich erschienen Editorial kritisierte H. Kehlet [7] den globalen Zielparameter Schmerzintensität und plädierte für eine (operationsspezifische) Messung der funktionsassoziierten Schmerzstärke. In eine ähnliche Richtung geht die konkrete Erfassung der Beeinträchtigung wichtiger postoperativer Funktionen sowie Nebenwirkungen, wie sie beispielsweise im QUIPS-Projekt mit einfachen Ja/ Nein-Fragen erfolgt [8]: Solche Parame- ter bzw. daraus abgeleitete Indizes können theoretisch ein Integral über Schmerzintensität, Funktion als auch Nebenwirkungen abbilden und damit dem Ziel eines umfassenden Qualitätsindikators in der Schmerzmedizin näherkommen. Allerdings muss dabei berücksichtigt werden, dass die Vorstellungen von Chirurgen, Pflegekräften, Patienten, Anästhesisten oder Klinikbetreibern über die Ziele von Schmerzmedizin - und dem, was unter guter Qualität zu verstehen sei erheblich differieren können. Auch dies spiegelt sich in der völlig konträren Einschätzung der Zertifizierungserfolge bei Pflegenden und Ärzten in der Arbeit von Böhmer et al. wider.

Der einzige Ausweg aus diesem Dilemma besteht nach Ansicht des Autors dieses Editorials in einem interprofessionell zu führenden Dialog, um einen Paradigmenwechsel in der schmerzmedizinischen Qualitätsdiskussion herbeizuführen: weg von der eindimensionalen Reduktion von Schmerzintensität (schon gar nicht auf den Wert „0“), hin zu einem breiter angelegten Qualitätsziel, das verschiedene Aspekte der postoperativen Rehabilitation umfasst - und damit auch nicht mehr Aufgabe einer einzelnen Fachoder Berufsgruppe ist. Ohne dieser Diskussion vorzugreifen, könnte ein solches Ziel beispielsweise wie folgt beschrieben werden: möglichst frühzeitige und komplikationslose Mobilisierung, Ernährung und Entlassung eines schmerzarmen und $z u$ friedenen Patienten.

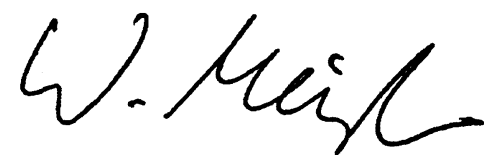

W. Meissner

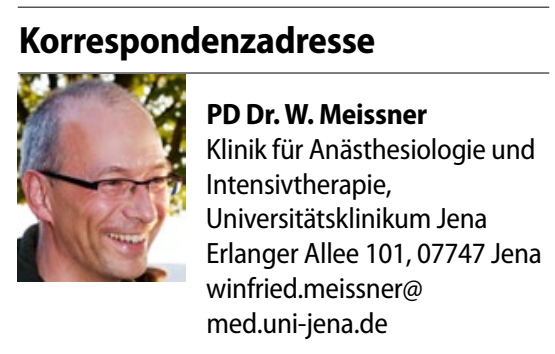

\section{Literatur}

1. Böhmer AB, Poels M, Simanski C et al (2012) Qualitätsmanagement in der Akutschmerztherapie. Ergebnisse einer Befragung TüV-zertifizierter Kliniken. Schmerz

2. Gould TH, Crosby DL, Harmer M et al (1992) Policy for controlling pain after surgery: effect of sequential changes in management. BMJ 305:1187-1193

3. Mularski RA, White-Chu F, Overbay D (2006) Measuring pain as the 5 th vital sign does not improve quality of pain management. J Gen Intern Med 21:607-612

4. Lucas CE, Vlahos AL, Ledgerwood AM (2007) Kindness kills: the negative impact of pain as the fifth vital sign. J Am Coll Surg 205:101-107

5. Meissner W, Mescha S, Rothaug J et al (2008) Qualitätsverbesserung in der postoperativen Schmerztherapie. Dtsch Arztebl 105:865-870

6. Zaslansky R, Chapman CR, Rothaug J et al (2012) Feasibility of International Data Collection and Feedback on Postoperative Pain Data: proof of concept. Eur J Pain 16:430-438

7. Kehlet H, Dahl JB (2011) Assessment of postoperative pain-need for action! Pain 152:1699-1700

8. Rothaug J, Weiss T, Meissner W (2012) How simple can it get? Measuring pain with NRS items or binary items. Clin J Pain (im Druck) 